February 3, 2019 12:48 WSPC/INSTRUCTION FILE $\quad$ ESCPO10

International Journal of Modern Physics A

(C) World Scientific Publishing Company

\title{
Validation of Transfer Map Calculation for Electrostatic Deflectors in the Code COSY INFINITY
}

\author{
Eremey Valetov; Martin Berz, Kyoko Makino \\ Department of Physics and Astronomy, Michigan State University \\ East Lansing, Michigan 48824, the United States of America
}

Received Day Month Year

Revised Day Month Year

\begin{abstract}
The beam physics code COSY INFINITY uses a beamline coordinate system with a Frenet-Serret frame relative to the reference particle, and calculates differential algebra-valued transfer maps by integrating the ODEs of motion in the respective vector space over a differential algebra (DA).

We described and performed computation of the DA transfer map of an electrostatic spherical deflector in a laboratory coordinate system using two conventional methods: (1) by integrating the ODEs of motion using a numerical integrator and (2) by computing analytically and in closed form the properties of the respective elliptical orbits from Kepler theory. We compared the resulting transfer maps with (3) the DA transfer map of COSY INFINITY's built-in electrostatic spherical deflector element ESP and (4) the transfer map of the electrostatic spherical deflector computed using the program GIOS, which uses analytic formulas from a paper ${ }^{1}$ by Hermann Wollnik regarding second order aberrations.

In addition to the electrostatic spherical deflector, we studied an electrostatic cylindrical deflector, where the Kepler theory is not applicable. We computed the DA transfer map by the ODE integration method (1), and we compared it with the transfer maps by (3) COSY INFINITY's built-in electrostatic cylindrical deflector element ECL and (4) GIOS.

The transfer maps of electrostatic spherical and cylindrical deflectors obtained using the direct calculation methods (1) and (2) are in excellent agreement with those computed using (3) COSY INFINITY. On the other hand, we found a significant discrepancy with (4) the program GIOS.
\end{abstract}

Keywords: Electrostatic deflectors; transfer maps; aberrations; tracking code; COSY INFINITY; differential algebra.

PACS numbers: 41.85.Gy, 41.85.-p, 29.30.-h, 41.20.Cv, 02.60.Cb, 29.90.+r

*valetove@msu.edu

${ }^{\dagger}$ Presently also affiliated with the Physics Department, Lancaster University, Bailrigg, Lancaster LA1 4YW, UK, as well as with the Cockcroft Institute of Accelerator Science and Technology, Daresbury International Science Park, Daresbury, Warrington WA4 4AD, UK. 


\section{Introduction}

Aberrations of electrostatic deflectors have to be taken into account in accelerator physics, where maintaining a small beam emittance or sufficient spin coherence is often an important consideration, as well as in spectrometry, where that is necessary for accurate ion beam analysis. In particular, this is vital for accurate modeling and simulations of storage rings with electrostatic deflectors, such as frozen spin ${ }^{2}$ and quasi-frozen $\operatorname{spin}^{3,4}$ lattices for measurement of the electrostatic dipole moment (EDM), especially that not accurately accounting for electrostatic aberrations provides a mechanism for energy conservation violation.

We were informed ${ }^{5}$ of a discrepancy between results for the second order electrostatic deflector aberration ${ }^{\mathrm{a}}(x \mid x a)$ calculated using the beam physics tracking code COSY INFINITY,${ }^{6}$ where aberrations are computed numerically using differentialalgebraic (DA) transfer map methods, and the program ${ }^{7}$ LISE $^{++}$, which uses aberration formulas from Hermann Wollnik's 1965 paper. ${ }^{1}$ We note that the program $G I O S^{8}$ also uses the electrostatic deflector aberration formulas from the mentioned paper by Wollnik.

In COSY INFINITY, the equations of motion are integrated, once for each particle optical element comprising the lattice, in phase space represented ${ }^{9(p p .86-100)}$ as a vector space $\left({ }_{n} D_{v}\right)^{v}$ over a differential algebra (DA) ${ }_{n} D_{v}$, where $n$ is the computation order and $v$ is the number of phase space coordinates. The result is a transfer map $\mathcal{M}$ that expresses the final coordinates $z_{\mathrm{f}}$ of any ray as $z_{\mathrm{f}}=\mathcal{M}\left(z_{\mathrm{i}}\right)$, where $z_{\mathrm{i}}$ are the initial coordinates ${ }^{9(\text { Chs. } 4-5)}$.

We performed a validation study of the DA transfer maps produced for electrostatic deflectors in COSY INFINITY by directly calculating the transfer maps for electrostatic spherical and cylindrical deflectors in the laboratory coordinate system (LCS). COSY INFINITY includes built-in electrostatic deflector particle optical elements: electrostatic spherical deflector ESP, electrostatic cylindrical deflector ECL, and inhomogeneous combined-function electrostatic elements ES and EC.

For the case of an electrostatic spherical deflector, we used two conventional methods to calculate the non-relativistic DA transfer map: (1) integration of the ODEs of motion using a fourth order Runge-Kutta integrator and (2) computation and application of Kepler theory's transition matrix with elements as the Lagrange coefficients. We compared the results with (3) the DA transfer map of the nonrelativistic version of COSY INFINITY's built-in electrostatic spherical deflector element ESP and (4) the transfer map of the electrostatic spherical deflector computed using the program GIOS.

In the case of an electrostatic cylindrical deflector, where the Kepler theory is not applicable, we computed and compared the transfer maps by the methods (1) integration of the ODEs in the LCS, (3) using COSY INFINITY's ECL, and (4)

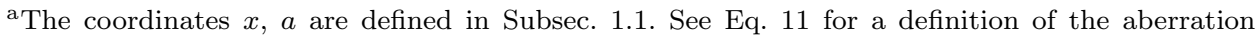
notation $(\ldots \mid \ldots)$. 
using GIOS.

We found excellent agreement between the transfer maps of electrostatic deflectors calculated using these methods in LCS and using COSY INFINITY, which validates COSY INFINITY for the purpose of calculating aberrations of electrostatic deflectors. On the other hand, we found a significant discrepancy for the aberration $(x \mid x a)$ with the program GIOS.

The validation of COSY INFINITY for electrostatic deflectors is supportive of our arguments in Ref. 10, where we derived analytic formulas for aberrations of the electrostatic deflector order by order using a perturbation method and compared the derived formulas with analytic formulas from Wollnik's paper, ${ }^{1}$ as well as with the code COSY INFINITY.

\subsection{COSY INFINITY's Beamline Coordinate System}

COSY INFINITY uses a beamline coordinate system that comprises the phase space coordinates ${ }^{11(\mathrm{p} .9)}$

$$
\begin{array}{ll}
r_{1}=x, & r_{2}=a=p_{x} / p_{0}, \\
r_{3}=y, & r_{4}=b=p_{y} / p_{0}, \\
r_{5}=l=-\left(t-t_{0}\right) v_{0} \frac{\gamma}{1+\gamma}, & r_{6}=\delta_{K}=\frac{K-K_{0}}{K} .
\end{array}
$$

Coordinates $x$ and $y$ are the transversal Frenet-Serret position coordinates in meters (with the $x$ axis antiparallel to the normal vector of the reference orbit by the beam physics convention), $p$ is the momentum, $K$ is the kinetic energy, $v$ is the velocity, $t$ is the time of flight, and $\gamma$ is the Lorentz factor. For the purposes of this study, $\gamma=1$. The index zero refers to the reference particle.

\subsection{Transfer Map Calculation by Integrating the ODEs in Laboratory Coordinates}

We considered a bunch of non-relativistic charged particles launched with kinetic energy $K_{0}=m v_{0}^{2} / 2$ and zero potential energy, where $m$ is the particle mass and $v_{0}$ is the reference velocity. We supposed a circular reference orbit of radius $r_{0}$ to be defined for the particle bunch in an electrostatic deflector. Following the convention of the potential energy as zero at the reference orbit, we calibrated the potential energy of the charged particles to

$$
U(r)=-\frac{\alpha}{r}+\frac{\alpha}{r_{0}}
$$

for the electrostatic spherical deflector and to

$$
U(r)=Z e V(r)=\alpha \log \frac{r}{r_{0}}
$$

for the electrostatic cylindrical deflector, where $\alpha$ can be expressed as $\alpha=Z e \chi_{\mathrm{e}} r_{0}$ in terms of the particle charge $q=Z e$, the reference orbit radius $r_{0}$, and the electric rigidity $\chi_{\mathrm{e}}=p v / Z e$. 
By energy conservation, an off-reference particle at initial radius $r_{\mathrm{i}}$ would upon entering the deflector have an initial velocity magnitude $v_{\mathrm{i}}$ such that

$$
v_{\mathrm{i}}=\sqrt{v_{0}^{2}-\frac{2}{m} Z e \chi_{\mathrm{e}}\left(1-\frac{r_{0}}{r_{\mathrm{i}}}\right)}
$$

for the electrostatic spherical deflector and

$$
v_{\mathrm{i}}=\sqrt{v_{0}^{2}-\frac{2}{m} Z e \chi_{\mathrm{e}} r_{0} \log \frac{r_{\mathrm{i}}}{r_{0}}} .
$$

for the electrostatic cylindrical deflector.

COSY INFINITY's horizontal transversal coordinate $x$ is defined relative to the circular reference orbit as $x=r-r_{0}$, where $r$ is the length of the projection of a particle's radius vector on the plane of the reference particle's orbital motion. COSY INFINITY's horizontal momentum component $a=p_{x} / p_{0}$ can be, in the non-relativistic case, expressed as $a=v_{x} / v_{0}$, where $v_{x}$ is the $x$ component of a particle's velocity and $v_{0}$ is the reference velocity.

Now, let $\left(x_{\mathrm{i}}, a_{\mathrm{i}}\right)$ be the initial beamline coordinates of the particle in the electrostatic spherical or cylindrical deflector. In reality, the deflector would have a fringe field; in this model, we approximate the fringe field by an instant jump in the electrostatic potential from zero to the electrostatic potential of the electrostatic deflector at radius $r_{\mathrm{i}}=r_{0}+x_{\mathrm{i}}$. Thus, all particles in a bunch experience a "step down" from kinetic energy $K_{0}$ to a kinetic energy $K=K_{0}-U(r)$ at time $t=0$.

In a similar way, at the end of the element, the particle will experience a "step up" due to the change of the potential energy back to zero. Note that these changes of energy are essential even in the absence of a true fringe field treatment to preserve the actual beam energy.

Considering the above, from the second Newton law and conservation of the angular momentum, the ODEs of motion in the polar laboratory coordinate system $(r, \theta)$ are

$$
\frac{d}{d \theta}\left(\begin{array}{c}
r \\
v_{r} \\
\theta \\
\omega
\end{array}\right)=\left(\begin{array}{c}
\frac{\frac{v_{r}}{\omega}}{-\frac{\mu}{r^{2}} \frac{1}{\omega}}+\omega r \\
1 \\
-2 \frac{h}{r^{3}} \frac{v_{r}}{\omega}
\end{array}\right)
$$

for the electrostatic spherical deflector and

$$
\frac{d}{d \theta}\left(\begin{array}{c}
r \\
v_{r} \\
\theta \\
\omega
\end{array}\right)=\left(\begin{array}{c}
\frac{v_{r}}{\omega} \\
-\frac{\mu}{r} \frac{1}{\omega}+\omega r \\
1 \\
-2 \frac{h}{r^{3}} \frac{v_{r}}{\omega}
\end{array}\right)
$$

for the electrostatic cylindrical deflector, where $\mu=\alpha / m, \omega$ is the angular velocity, and $h=\omega r^{2}=|\vec{r} \times \vec{v}|$ is the massless angular momentum.

We calculated the DA transfer maps of the electrostatic spherical and cylindrical deflectors, with conversion from polar $(r, \theta)$ to $(x, a)$ beamline coordinates, by 
integration of the ODEs of motion in polar laboratory coordinates using a fourth order Runge-Kutta integrator ${ }^{\mathrm{b}}$. The reference orbit radius $r_{0}=1 \mathrm{~m}$ was used.

To obtain a DA-valued transfer map, in both spherical and cylindrical deflector cases we set the initial phase space coordinates as

$$
\left(x_{\mathrm{i}}, a_{\mathrm{i}}\right)=\left(d_{1}, d_{2}\right)=(\mathrm{DA}(1), \mathrm{DA}(2)),
$$

the respective DA generators ${ }^{9(\mathrm{pp} .86-96)}$. We performed the calculations with the computation order three.

\subsection{Transfer Map Calculation for an Electrostatic Spherical Deflector Using Lagrange Coefficients}

Motion in a central field with a potential energy of the form $U(r)=-\alpha / r+$ const is described by conventional Kepler theory; in particular, by the equation of orbit

$$
r=\frac{p}{1+e \cos f}
$$

where $p$ is the focal parameter, $e$ is the orbit eccentricity, and polar angle $f$ is called the true anomaly ${ }^{12(\mathrm{p} .117)}$. True anomaly $f=0$ corresponds to the direction of the perihelion, that is, the point of the orbit nearest to the orbital focus at the origin of the polar coordinate system $(r, f)$. Let $\theta=f_{\mathrm{f}}-f_{\mathrm{i}}$ be the true anomaly difference between the final and initial positions.

The particle position $\vec{r}$ is expressed as

$$
\vec{r}=\vec{i}_{e} r \cos f+\vec{i}_{p} r \sin f,
$$

in terms of polar coordinates $(r, f)$ and the basis vectors $\left(\vec{i}_{e}, \vec{i}_{p}\right)$ of the heliocentric coordinate system, where $\vec{i}_{e}$ is a unit vector in the direction of perihelion and unit vector $\vec{i}_{p}$ is chosen so that $\vec{i}_{e} \times \vec{i}_{p}$ is codirectional with the vector of the angular velocity of the particle, as Fig. 1 illustrates.

Kepler theory's transition matrix

$$
\Phi=\left(\begin{array}{cc}
F & G \\
F_{t} & G_{t}
\end{array}\right)
$$

expresses the final coordinates $\left(\vec{r}_{\mathrm{f}}, \vec{v}_{\mathrm{f}}\right)$ as a function of the initial coordinates $\left(\vec{r}_{\mathrm{i}}, \vec{v}_{\mathrm{i}}\right)$ as

$$
\left(\begin{array}{c}
\vec{r}_{\mathrm{f}} \\
\vec{v}_{\mathrm{f}}
\end{array}\right)=\Phi\left(\begin{array}{c}
\vec{r}_{\mathrm{i}} \\
\vec{v}_{\mathrm{i}}
\end{array}\right)
$$

\footnotetext{
${ }^{\mathrm{b}}$ We had also performed numerical integration of the ODEs of motion using an eighth order Runge-Kutta-Verner integrator with adaptive stepsize and obtained similar results; however, source code for a fourth order Runge-Kutta integrator is shorter and simpler for redistribution.
} 


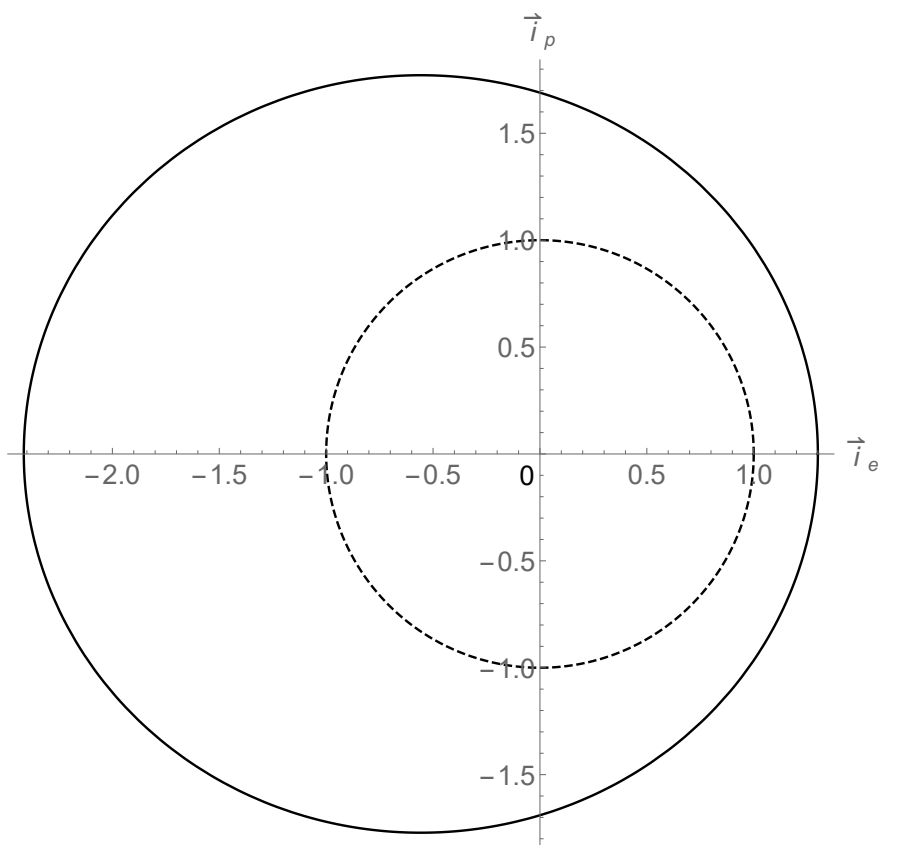

Fig. 1. Orbit of a particle launched counter-clockwise at polar angle $f_{\mathrm{i}}=0$ with initial beamline coordinates $\left(x_{\mathrm{i}}, a_{\mathrm{i}}\right)=(0.3 \mathrm{~m}, 0)$ through an electrostatic spherical deflector (solid ellipse). The reference orbit (dashed circle) has the radius $r_{0}=1 \mathrm{~m}$. The plot illustrates the basis vectors $\left(\vec{i}_{e}, \vec{i}_{p}\right)$ of the heliocentric coordinate system in relation to the orbit geometry.

and comprises elements ${ }^{12(p p .128-131)}$

$$
\begin{aligned}
F(\theta) & =1-\frac{r_{\mathrm{f}}}{p}(1-\cos \theta), \\
F_{t}(\theta) & =\frac{\sqrt{\mu}}{r_{\mathrm{i}} p}\left[\sigma_{0}(1-\cos \theta)-\sqrt{p} \sin \theta\right], \\
G(\theta) & =\frac{r_{\mathrm{f}} r_{\mathrm{i}}}{\sqrt{\mu p}} \sin \theta, \\
G_{t}(\theta) & =1-\frac{r_{\mathrm{i}}}{p}(1-\cos \theta) .
\end{aligned}
$$

The elements $F, F_{t}, G$, and $G_{t}$ of the transition matrix $\Phi$ are called Lagrange coefficients. The Lagrange coefficients $F_{t}$ and $G_{t}$ are simply time derivatives of $F$ and $G$, respectively.

It can be shown that the focal parameter $p$ and the eccentricity $e$ depend only on reference orbit radius $r_{0}$ and initial beamline coordinates $\left(x_{\mathrm{i}}, a_{\mathrm{i}}\right)$, and we have done that in the course of our work on this topic.

We calculated the DA transfer map of the electrostatic spherical deflector, with conversion from polar $(r, \theta)$ to $(x, a)$ beamline coordinates, using Kepler theory's transition matrix with elements as the Lagrange coefficients in terms of the true 
anomaly difference. The reference orbit radius $r_{0}=1 \mathrm{~m}$ was used. We performed the calculations with the computation order three.

\section{Transfer Maps of an Electrostatic Spherical Deflector and Comparison}

Here, we list and compare the DA transfer maps for particles passing through a $45^{\circ}$ sector of the electrostatic spherical deflector, calculated

(1) by integration of the ODEs of motion in polar laboratory coordinates (Eq. 5) using a fourth order Runge-Kutta integrator;

(2) using the transition matrix with elements as the Lagrange coefficients in terms of the true anomaly difference;

(3) for COSY INFINITY's built-in electrostatic spherical deflector element ESP; and

(4) using the code sequence E S in the program GIOS.

In all cases, the reference orbit radius is $r_{0}=1 \mathrm{~m}$, and non-relativistic equations of motion were used. For definiteness, the particles in the bunch were set to kinetic energy $1 \mathrm{MeV}$, mass $1 \mathrm{amu}$, and charge $1 e$; however, as noted above, this setting has no impact on the orbit geometry as long as the reference orbit radius is kept the same by adjusting the voltages of the inner and outer spherical shells of the electrostatic spherical deflector.

For visual transfer map comparison purposes, the computation order three was used in all cases, except for GIOS. The code GIOS uses momentum-like coordinates that differ from those of COSY INFINITY, which manifests itself starting from the order three in terms of $x$ and $a$. Thus, we only used the computation order two in calculations using GIOS, whereas the computation order three would not have been directly useful for the comparison ${ }^{\mathrm{c}}$.

The Jacobian $M=\operatorname{Jac}(\mathcal{M})$ of the transfer map $\mathcal{M}$ of any Hamiltonian system satisfies the symplecticity condition ${ }^{9(\mathrm{pp} .155-159)} M \cdot \hat{J} \cdot M^{\mathrm{T}}=\hat{J}$, where the phase space coordinates are ordered as $z=\left(q_{1}, \ldots, q_{m}, p_{1}, \ldots, p_{m}\right),\left(q_{1}, \ldots, q_{m}\right)$ are the canonical position coordinates and $\left(p_{1}, \ldots, p_{m}\right)$ are the conjugate momentum coordinates,

$$
\hat{J}=\left(\begin{array}{cc}
0 & I_{m} \\
-I_{m} & 0
\end{array}\right),
$$

$I_{m}$ is an $m \times m$ identity matrix, and $m$ is the phase space dimension.

${ }^{\mathrm{c}}$ We note that GIOS has a relatively new output mode that uses $a=p_{x} / p_{0}$ and $b=p_{y} / p_{0}$ as momentum-like coordinates. This mode can be selected using the command S C. First and second order transfer map elements in this mode are the same as when expressed in GIOS's default coordinates. Additionally, first and second order transfer map elements printed in Transport ${ }^{13}$ and GIOS coordinates by COSY INFINITY's command PT are the same as those printed in COSY INFINITY coordinates by COSY INFINITY's command PM (up to a trivial unit conversion that applies in this specific context). 
For each transfer map calculation case, we computed deviations from the conditions of symplecticity ${ }^{9(p p .155-159), 14}$ for the first and second order aberration coefficients and motion in the $x$ - $a$ plane. These conditions of symplecticity are as follows:

$$
\begin{aligned}
& g_{1}=(x \mid x)(a \mid a)-(a \mid x)(x \mid a)-1=0, \\
& g_{2}=(x \mid x)(a \mid x a)-(a \mid x)(x \mid x a)+(x \mid x x)(a \mid a)-(a \mid x x)(x \mid a)=0, \\
& g_{3}=(x \mid x)(a \mid a a)-(a \mid x)(x \mid a a)+(x \mid x a)(a \mid a)-(a \mid x a)(x \mid a)=0,
\end{aligned}
$$

where the aberration coefficient $\left(z_{i} \mid z_{j_{1}} \cdots z_{j_{n}}\right)$ is the partial derivative

$$
\left(z_{i} \mid z_{j_{1}} \cdots z_{j_{n}}\right)=\left(\frac{\partial^{n}(\mathcal{M}(z))_{i}}{\partial z_{j_{1}} \cdots \partial z_{j_{n}}}\right)_{z=0}
$$

of the $i$-th component of the respective transfer map $\mathcal{M}$ applied to a coordinate vector $z=\left(z_{1} \cdots z_{2 m}\right)$, and $2 m$ is the number of phase space coordinates.

\subsection{Transfer Map Obtained by Integrating the ODEs in

\begin{tabular}{|c|c|c|c|}
\hline \multicolumn{4}{|l|}{$\mathrm{X}_{-} \mathrm{f}$} \\
\hline & I COEFFICIENT & ORDER & EXPONENTS \\
\hline & 0.7071067811865617 & 1 & $100 \quad 0$ \\
\hline & 0.7071067811865485 & 1 & $0 \quad 1$ \\
\hline & -.4999999999998140 & 2 & 20 \\
\hline & 0.9999999999999932 & 2 & 11 \\
\hline & 0.2071067811865112 & 2 & $0 \quad 2$ \\
\hline & -.3535533905929528 & 3 & 30 \\
\hline & $0.6066017177973747 \mathrm{E}-01$ & 3 & 12 \\
\hline & 0.2928932188133920 & 3 & 03 \\
\hline \multicolumn{4}{|l|}{$A_{-} f$} \\
\hline & COEFFICIENT & ORDER & EXPONENTS \\
\hline & -.7071067811864190 & 1 & $100 \quad 0$ \\
\hline & 0.7071067811865486 & 1 & $0 \quad 1$ \\
\hline & -.7071067811865216 & 2 & 02 \\
\hline & -.3535533905931392 & 3 & 30 \\
\hline & -1.060660171779751 & 3 & 12 \\
\hline
\end{tabular} Laboratory Coordinates}

The transfer map from the integration of the ODEs of motion in polar laboratory coordinates using a fourth order Runge-Kutta integrator is shown in Listing 2.1.

Listing 2.1: Third order transfer map for $x$ - $a$ motion in the electrostatic spherical deflector, obtained in laboratory coordinates by integration of the ODEs of motion using a fourth order Runge-Kutta integrator. DA coefficients with absolute values less than $10^{-11}$ are omitted.

The deviations $g_{1}, g_{2}$, and $g_{3}$ from the conditions of symplecticity listed in Eq. 10 in this case were as follows:

$$
\begin{aligned}
& g_{1}=-0.7949196856316121 \times 10^{-13}, \\
& g_{2}=-0.2514743315828557 \times 10^{-12}, \\
& g_{3}=-0.1188442801551391 \times 10^{-12} .
\end{aligned}
$$


February 3, 2019 12:48 WSPC/INSTRUCTION FILE ESCPO10

Validation of Transfer Map Calculation for Electrostatic Deflectors in the Code COSY INFINITY 9

\subsection{Transfer Map Obtained Using Lagrange Coefficients in Laboratory Coordinates}

The transfer map obtained in laboratory coordinates using the Lagrange coefficients transition matrix is shown in Listing 2.2 .

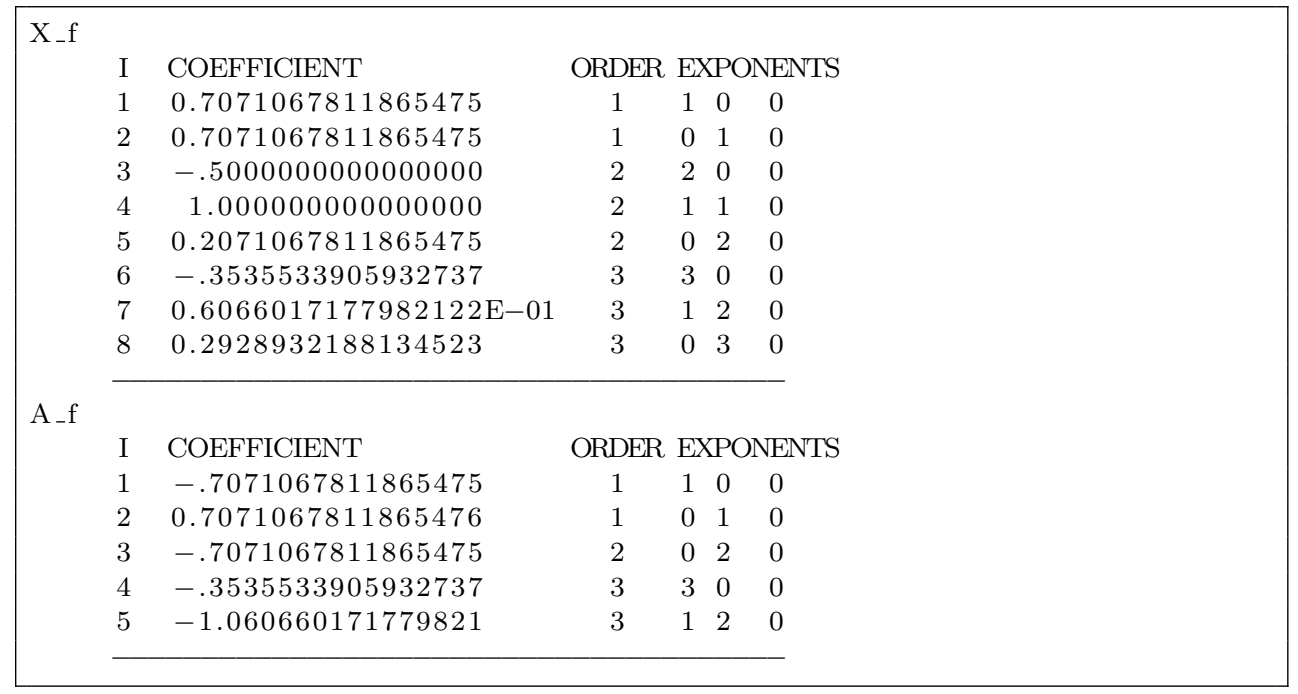

Listing 2.2: Third order transfer map for $x$ - $a$ motion in the electrostatic spherical deflector, obtained in laboratory coordinates using Kepler theory's transition matrix with elements as Lagrange coefficients. DA coefficients with absolute values less than $10^{-11}$ are omitted.

The deviations $g_{1}, g_{2}$, and $g_{3}$ from the conditions of symplecticity listed in Eq. 10 in this case were as follows:

$$
\begin{aligned}
& g_{1}=-0.1110223024625157 \times 10^{-15}, \\
& g_{2}=0.3119771259853192 \times 10^{-16}, \\
& g_{3}=0.1110223024625157 \times 10^{-15}
\end{aligned}
$$

\subsection{Transfer Map of COSY INFINITY's Built-In Electrostatic Spherical Deflector Element ESP}

The transfer map of COSY INFINITY's built-in electrostatic spherical deflector element ESP, obtained using non-relativistic equations of motion, is shown in Listing 2.3 .

The deviations $g_{1}, g_{2}$, and $g_{3}$ from the conditions of symplecticity listed in Eq. 10 were as follows:

$$
\begin{aligned}
& g_{1}=-0.2220446049250313 \times 10^{-15}, \\
& g_{2}=0.2220446049250313 \times 10^{-15}, \\
& g_{3}=0.3330669073875470 \times 10^{-15} .
\end{aligned}
$$

We note that for simplicity, essentially the same result can be obtained using the relativistic equations of motion that are used by default in COSY INFINITY by 


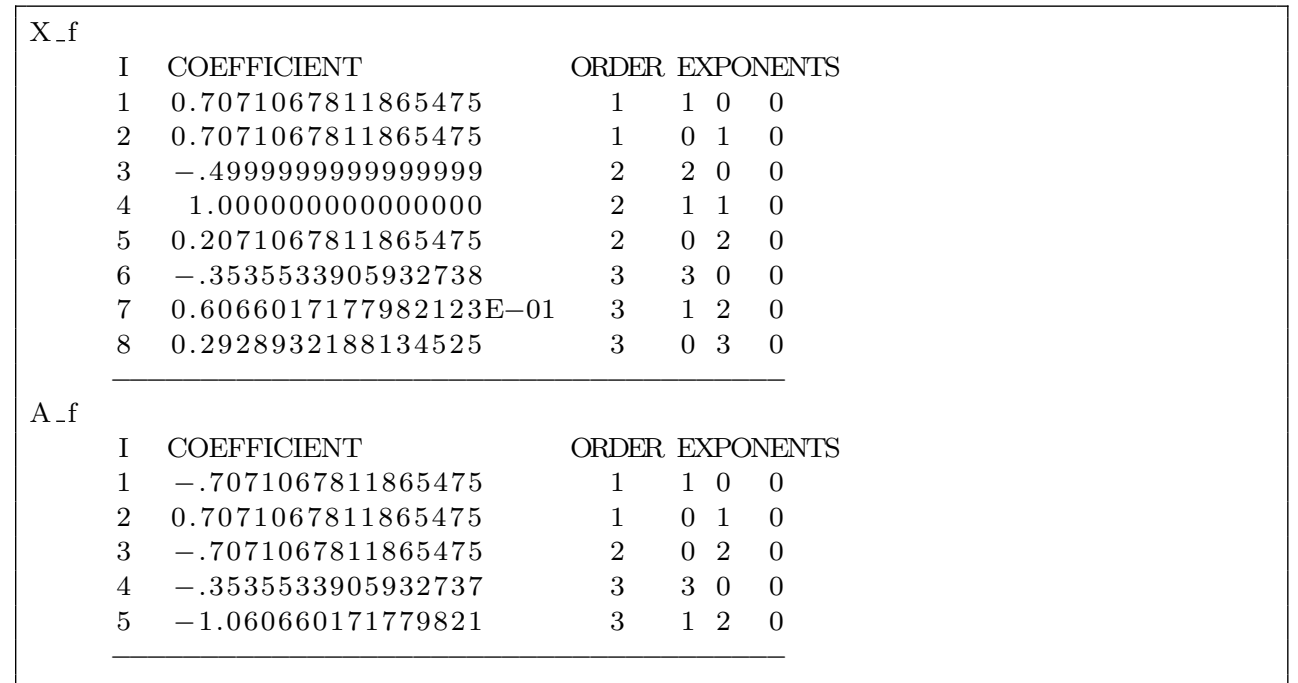

Listing 2.3: Third order transfer map for $x$ - $a$ motion in the electrostatic spherical deflector, obtained using COSY INFINITY's built-in electrostatic spherical deflector element ESP, with nonrelativistic equations of motion.

simply using a very low kinetic energy for the calculation. For example, the transfer map computed using the relativistic equations with the kinetic energy $10^{-7} \mathrm{~m}$ agrees with the above-listed transfer map to about $10^{-10}$. In this case, the values of the deviations $g_{1}, g_{2}$, and $g_{3}$ are $\sim 10^{-15}$.

\subsection{Transfer Map Computed Using the Electrostatic Spherical Deflector Element in the Program GIOS}

The second order transfer map of the electrostatic spherical deflector computed using the code sequence E S in the program GIOS is shown in Listing 2.4.

The deviations $g_{1}, g_{2}$, and $g_{3}$ from the conditions of symplecticity listed in Eq. 10 were as follows:

$$
\begin{aligned}
& g_{1}=0.3804934145534844 \times 10^{-10}, \\
& g_{2}=-0.2928932188380493, \\
& g_{3}=0.7071067812000000 .
\end{aligned}
$$

We note that the deviations $g_{2}$ and $g_{3}$ are significant in magnitude and indicate error(s) in the program GIOS.

We also note that these differences are not due to the fact that GIOS uses momentum-like coordinates that differ from those of COSY INFINITY. The respective effects manifest themselves only in order three in $x$ and $a$ terms, which we are not comparing here. 


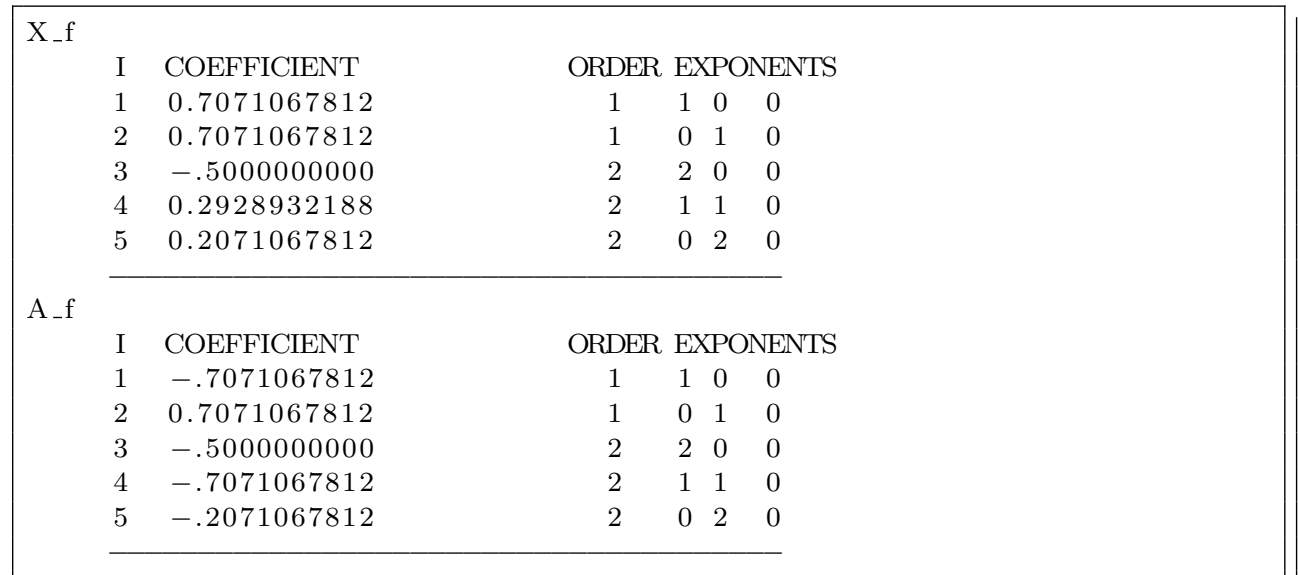

Listing 2.4: Second order transfer map for $x$ - $a$ motion in the electrostatic spherical deflector, obtained using the code sequence $\mathrm{E} \mathrm{S}$ in the program GIOS.

\section{Transfer Maps of an Electrostatic Cylindrical Deflector and Comparison}

In this section, we list and compare the DA transfer maps for particles passing through a $45^{\circ}$ sector of the electrostatic cylindrical deflector, calculated

(1) by integration of the ODEs of motion in polar laboratory coordinates (Eq. 6) using a fourth order Runge-Kutta integrator;

(2) for COSY INFINITY's built-in electrostatic cylindrical deflector element ECL; and

(3) using the code sequence E S in the program GIOS.

In all cases, the reference orbit radius is $r_{0}=1 \mathrm{~m}$, and non-relativistic equations of motion were used. For definiteness, the particles in the bunch were set to kinetic energy $1 \mathrm{MeV}$, mass $1 \mathrm{amu}$, and charge $1 e$; however, this setting has no impact on the orbit geometry as long as the reference orbit radius is kept the same by adjusting the voltages of the inner and outer cylindrical shells of the electrostatic cylindrical deflector. For visual transfer map comparison purposes, the computation order three was used in all cases, except for GIOS, where the computation order two was used.

\subsection{Transfer Map Obtained by Integrating the ODEs in Laboratory Coordinates}

The transfer map from the integration of the ODEs of motion in polar laboratory coordinates using a fourth order Runge-Kutta integrator is shown in Listing 3.1.

The deviations $g_{1}, g_{2}$, and $g_{3}$ from the conditions of symplecticity listed in Eq. 10 
February 3, 2019 12:48 WSPC/INSTRUCTION FILE ESCPO10

12 Eremey Valetov, Martin Berz, and Kyoko Makino

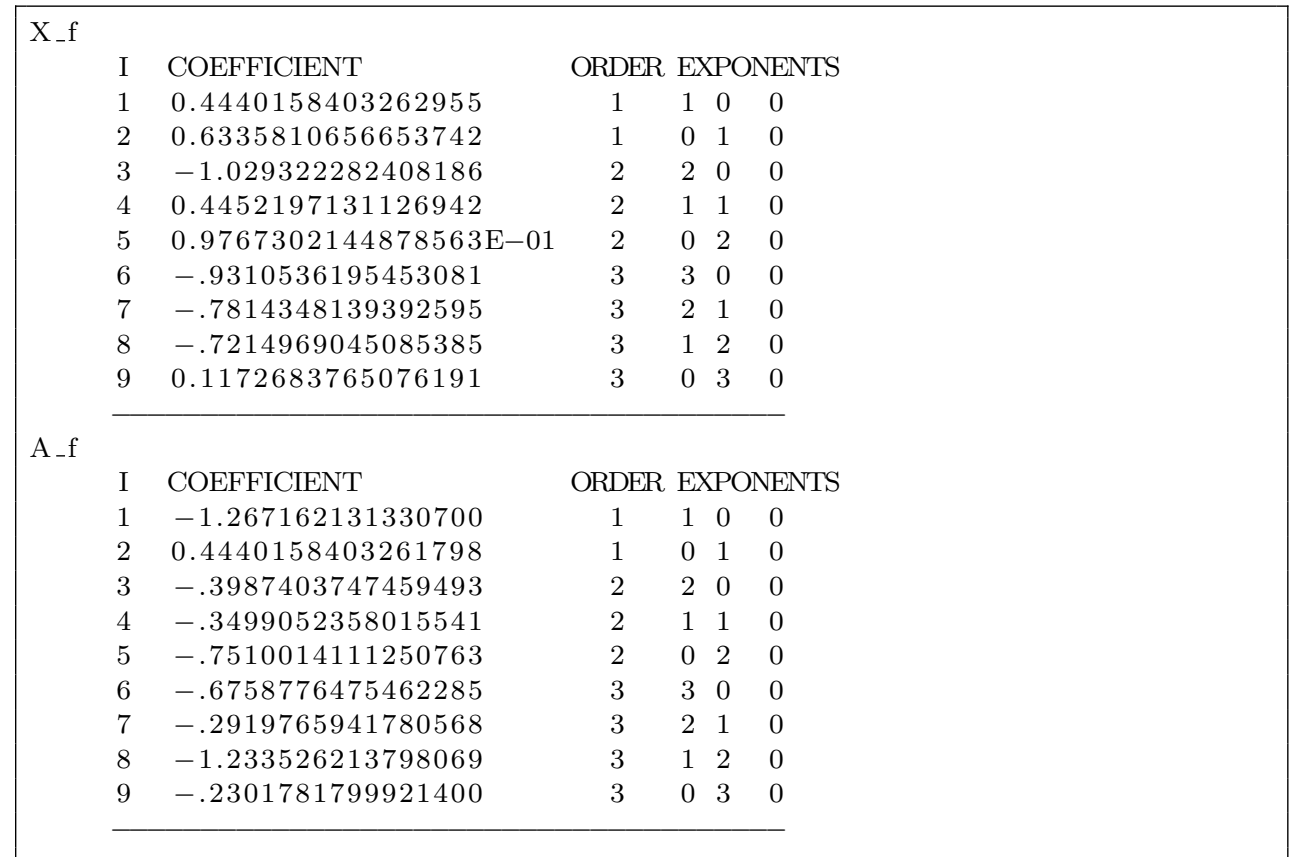

Listing 3.1: Third order transfer map for $x$ - $a$ motion in the electrostatic cylindrical deflector, obtained in laboratory coordinates by integration of the ODEs of motion using a fourth order Runge-Kutta integrator.

in this case were as follows:

$$
\begin{aligned}
& g_{1}=-0.7316369732279782 \times 10^{-13}, \\
& g_{2}=0.1615374500829603 \times 10^{-12}, \\
& g_{3}=-0.2078615057854449 \times 10^{-12} .
\end{aligned}
$$

\subsection{Transfer Map of COSY INFINITY's Built-In Electrostatic Cylindrical Deflector Element ECL}

The transfer map of COSY INFINITY's built-in electrostatic cylindrical deflector element ECL, obtained using non-relativistic equations of motion, is shown in Listing 3.2 .

The deviations $g_{1}, g_{2}$, and $g_{3}$ from the conditions of symplecticity listed in Eq. 10 were as follows:

$$
\begin{aligned}
& g_{1}=0.4440892098500626 \times 10^{-15}, \\
& g_{2}=0.2220446049250313 \times 10^{-15}, \\
& g_{3}=0.2498001805406602 \times 10^{-15} .
\end{aligned}
$$

We note that for simplicity, essentially the same result can be obtained using the relativistic equations of motion that are by default used in COSY INFINITY by simply using a very low kinetic energy for the calculation. For example, the transfer map computed using the relativistic equations with the kinetic energy $10^{-7} \mathrm{~m}$ agrees 


\begin{tabular}{|c|c|c|c|}
\hline \multicolumn{4}{|l|}{$X_{-} f$} \\
\hline I & COEFFICIENT & ORDER & EXPONENTS \\
\hline 1 & 0.4440158403262133 & 1 & $\begin{array}{lll}1 & 0 & 0\end{array}$ \\
\hline 2 & 0.6335810656653997 & 1 & $\begin{array}{ll}0 & 1\end{array}$ \\
\hline 3 & -1.029322282408272 & 2 & 20 \\
\hline 4 & 0.4452197131126671 & 2 & 11 \\
\hline 5 & $0.9767302144879608 \mathrm{E}-01$ & 2 & $0 \quad 2$ \\
\hline 6 & -.9310536195454117 & 3 & 30 \\
\hline 7 & -.7814348139394898 & 3 & 21 \\
\hline 8 & -.7214969045085790 & 3 & 12 \\
\hline 9 & 0.1172683765076182 & 3 & 03 \\
\hline \multicolumn{4}{|l|}{ A_f } \\
\hline I & COEFFICIENT & ORDER & EXPONENTS \\
\hline 1 & -1.267162131330799 & 1 & $1 \quad 0 \quad 0$ \\
\hline 2 & 0.4440158403262133 & 1 & 01 \\
\hline 3 & -.3987403747459333 & 2 & 20 \\
\hline 4 & -.3499052358016756 & 2 & 11 \\
\hline 5 & -.7510014111251326 & 2 & 02 \\
\hline 6 & -.6758776475462280 & 3 & 30 \\
\hline 7 & -.2919765941781459 & 3 & 21 \\
\hline 8 & -1.233526213798173 & 3 & 12 \\
\hline 9 & -.2301781799921575 & 3 & $0 \quad 3$ \\
\hline
\end{tabular}

Listing 3.2: Third order transfer map for $x$ - $a$ motion in the electrostatic cylindrical deflector, obtained using COSY INFINITY's built-in electrostatic cylindrical deflector element ECL, with non-relativistic equations of motion.

with the above listed transfer map to about $10^{-10}$. In this case, the values of the deviations $g_{1}, g_{2}$, and $g_{3}$ are $\sim 10^{-15}$.

\subsection{Transfer Map Computed Using the Electrostatic Cylindrical Deflector Element in the Program GIOS}

The second order transfer map of the electrostatic cylindrical deflector computed using the code sequence $\mathrm{E} \mathrm{S}$ in the program GIOS is shown in Listing 3.3.

The deviations $g_{1}, g_{2}$, and $g_{3}$ from the conditions of symplecticity listed in Eq. 10 were as follows:

$$
\begin{aligned}
& g_{1}=0.1705231511550664 \times 10^{-9}, \\
& g_{2}=-0.5559841747496004 \\
& g_{3}=0.6335810760905867
\end{aligned}
$$

We note that the deviations $g_{2}$ and $g_{3}$ are significant in magnitude and indicate error(s) in the program GIOS.

We also note that these differences are not due to the fact that GIOS uses momentum-like coordinates that differ from those of COSY INFINITY. The respective effects manifest themselves only in order three in $x$ and $a$ terms, which we are not comparing here. 


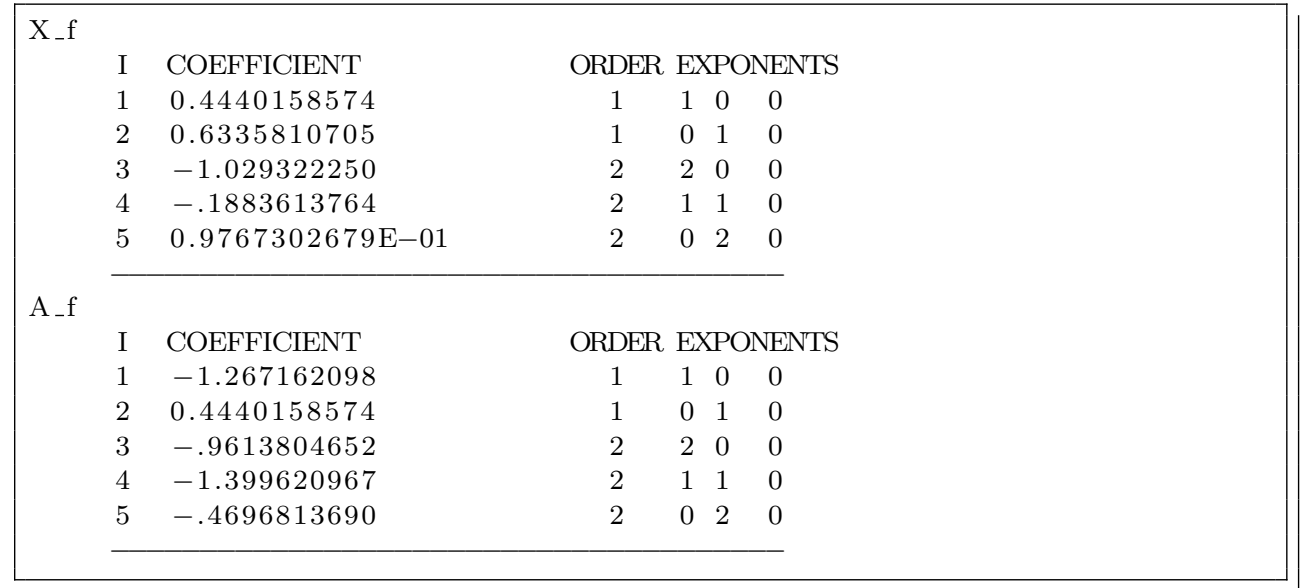

Listing 3.3: Second order transfer map for $x$ - $a$ motion in the electrostatic cylindrical deflector, obtained using the code sequence $\mathrm{E} \mathrm{S}$ in the program GIOS.

\section{Calculation Results, Comparison, and Conclusion}

The electrostatic spherical deflector transfer maps calculated in laboratory coordinates by integration of the ODEs and using the Lagrange coefficients transition matrix are in very high agreement with the transfer map of COSY INFINITY's built-in electrostatic spherical deflector element ESP. The deviations from the conditions of symplecticity $g_{1}, g_{2}$, and $g_{3}$ were the highest at $\sim 10^{-13}$ in case of integration of the ODEs in polar laboratory coordinates, the lowest at $\sim 10^{-16}$ in case of calculation using the Lagrange coefficients, and at $\sim 10^{-16}$ in case of the built-in element ESP.

The transfer map of the electrostatic spherical deflector computed using the program GIOS significantly disagrees with the other three transfer maps. However, the deviations $g_{2}$ and $g_{3}$ in the GIOS case were also significant in magnitude and indicate that the disagreement is due to error(s) in GIOS.

We arrived at an equivalent conclusion for the electrostatic cylindrical deflector transfer maps. The transfer map obtained by integrating the ODEs in laboratory coordinates agrees well with those obtained by COSY INFINITY's built-in electrostatic cylindrical deflector element ECL, both satisfying the symplectic conditions. On the other hand, the transfer map obtained using GIOS significantly disagrees with the other two transfer maps, and it significantly deviated from the symplecticity condition.

The results of this study validate COSY INFINITY's computation of DA transfer maps for electrostatic deflectors. We used this validation of COSY INFINITY in Ref. 10 to cross-validate the analytic formulas for electrostatic deflector aberrations that we derived there. 


\section{Electronic Supplementary Material}

The Reprint Server of Michigan State University's Center for Beam Theory and Dynamical Systems contains code to compute all test cases discussed in this paper on the webpage Ref. 15. This supplementary material can be also found by searching online for "ESCPO10".

\section{Acknowledgment}

This work was supported by the U.S. Department of Energy under Contract No. DE-FG02-08ER41546.

\section{References}

1. H. Wollnik, Second Order Approximation of the Three-Dimensional Trajectories of Charged Particles in Deflecting Electrostatic and Magnetic Fields, Nucl. Instrum. Methods 34, 213 (1965).

2. D. Anastassopoulos et al., AGS Proposal: Search for a Permanent Electric Dipole Moment of the Deuteron Nucleus at the $10^{-29}$ e.cm Level, BNL Report, Brookhaven National Laboratory, Upton, NY (2008).

3. Y. Senichev, S. Andrianov, M. Berz, S. Chekmenev, A. Ivanov, A. Lehrach, B. Lorentz, R. Maier, and E. Valetov, Quasi-Frozen Spin Method for EDM Deuteron Search, in Proc. 6th Int. Particle Accelerator Conf. (IPAC 2015), eds. S. Henderson, T. Satogata, and V. R. W. Schaa (JACoW, Geneva, 2015), MOPWA044.

4. E. Valetov, Ph.D. thesis, Fermilab report FERMILAB-THESIS-2017-21, Department of Physics and Astronomy, Michigan State University, East Lansing, MI 48824 (2017).

5. O. Tarasov, private communication.

6. K. Makino and M. Berz, COSY INFINITY Version 9, Nucl. Instrum. Methods A $\mathbf{5 5 8}$ (1), 346 (2006).

7. M. P. Kuchera, O. B. Tarasov, D. Bazin, B. M. Sherrill, and K. V. Tarasova, Plans for Performance and Model Improvements in the LISE ${ }^{++}$Software, Nucl. Instrum. Methods B 376, 168 (2016).

8. H. Wollnik, J. Brezina, and M. Berz, GIOS-BEAMTRACE - A Program Package to Determine Optical Properties of Intense Ion Beams, Nucl. Instrum. Methods A 258 (3), 408 (1987).

9. M. Berz, Modern Map Methods in Particle Beam Physics, Advances in Imaging and Electron Physics, Vol. 108 (Academic Press, San Diego, 1999).

10. E. Valetov and M. Berz, Derivation, Cross-Validation, and Comparison of Analytic Formulas for Electrostatic Deflectors, to appear in Advances in Imaging and Electron Physics.

11. M. Berz and K. Makino, COSY INFINITY 10.0 Beam Physics Manual, MSU Report MSUHEP 151103-rev, Department of Physics and Astronomy, Michigan State University, East Lansing, MI 48824 (2017).

12. R. H. Battin, An Introduction to the Mathematics and Methods of Astrodynamics, Revised Edition, rev. edn. (American Institute of Aeronautics and Astronautics, Reston, VA, 1999).

13. K. L. Brown, The Ion Optical Program TRANSPORT, Technical Report 91, SLAC National Accelerator Laboratory, Menlo Park, CA (1979).

14. H. Wollnik and M. Berz, Relations Between Elements of Transfer Matrices Due to the Condition of Symplecticity, Nucl. Instrum. Methods A 238 (1), 127 (1985). 
February 3, 2019 12:48 WSPC/INSTRUCTION FILE $\quad$ ESCPO10

Eremey Valetov, Martin Berz, and Kyoko Makino

15. E. Valetov, M. Berz, and K. Makino, Validation of Transfer Map Calculation for Electrostatic Deflectors in the Code COSY INFINITY (supplementary electronic resources). <https://bt.pa.msu.edu/cgi-bin/display.pl?name=ESCPO10 $>$. 